\title{
Quantitative evaluation of bone and cartilage changes after the Ravitch thoracoplasty by multislice computed tomography with 3-dimensional reconstruction
}

\author{
Pei-Yeh Chang, ${ }^{a}$ Jin-Yao Lai, ${ }^{a}$ Jeng-Chang Chen, ${ }^{a}$ and Chao-Jan Wang ${ }^{b}$
}

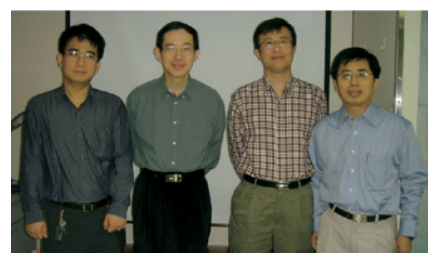

Drs Chen, Chang, Lai, and Wang (left to right)
Objective: Various bone and cartilage changes after the Ravitch thoracoplasty have recently been reported. In this study, quantitative measurements of long-term changes in the bone, cartilage, and contour of the chest wall through the use of multislice computed tomography with 3-dimensional reconstruction are presented.

Methods: Between 1985 and 2002, 114 patients with pectus excavatum received the Ravitch thoracoplasty in our hospital. Multislice computed tomography with 3-dimensional reconstruction was undertaken in 36 patients 4 to 18 years after the initial operation. Twenty-four patients with pectus excavatum without a surgical intervention were enrolled as a control group. The voxel size of calcification/ ossification in regenerated cartilage, costal cartilage shortening ratio, and clubbing index were calculated to quantify the long-term bone and cartilage changes.

Results: The calcification/ossification volume was measured as $5.29 \pm 6.94 \mathrm{~cm}^{3}$, which was significantly correlated with the patient's age at the time of the operation $(P=.001)$. The costal cartilage shortening ratio showed significant shortening of the regenerated cartilage in the third to sixth ribs bilaterally $(P<0.05)$, and the clubbing index confirmed significant clubbing in the bony ends of the third to fifth ribs $(P<0.05)$. These changes were not demonstrated in the control group.

Conclusions: After undergoing the Ravitch thoracoplasty, patients sustained significant bone and cartilage changes. The use of multislice computed tomography with 3-dimensional reconstruction may offer both images and quantitative measurements of these changes, but further investigation is necessary to elucidate the clinical impact of these phenomena.

$\mathrm{W}$ e demonstrated the occurrence of specific changes in the chest wall after the Ravitch thoracoplasty (RTP) through the use of modern multislice computed tomography (CT) with 3-dimensional reconstruction. ${ }^{1}$ Our gery $^{\mathrm{a}}$ and Radiology, ${ }^{\mathrm{b}}$ Chang Gung Children's Hospital, Chang Gung University, College of Medicine, Taoyuan, Taiwan.

Received for publication Dec 10, 2006; revisions received April 29, 2007; accepted for publication May 11, 2007.

Address for reprints: Chao-Jan Wang, MD, Department of Radiology, Chang Gung Children's Hospital, 5, Fu-Hsing St., Kweishan, Taoyuan 333, Taiwan (E-mail: cjwang@ adm.cgmh.org.tw).

J Thorac Cardiovasc Surg 2007;134:1279-83

0022-5223/\$32.00

Copyright (C) 2007 by The American Association for Thoracic Surgery

doi:10.1016/j.jtcvs.2007.05.067 previous studies showed a variety of changes in regenerated ribs, including missing or threadlike hypoplastic costal cartilage; clubbing, shortening, and constrictive changes to the ribs; and calcification/ossification $(\mathrm{C} / \mathrm{O})$ of regenerated cartilage. ${ }^{1}$ In the present study, these descriptive findings were further evaluated by quantitative measurement.

\section{Patients and Methods}

We attempted follow-up examinations in 114 consecutive patients with pectus excavatum who underwent RTP at Chang Gung Children's Hospital between 1985 and 2000. In addition, 24 patients who were candidates for corrective pectus excavatum surgery during 2005 were also included in this study as a control group. We arranged multislice CT with 3-dimensional reconstruction for these patients. Informed consent was obtained from all patients and their guardians, and this study was approved by the Ethics Committee of Chang Gung Memorial Hospital (IRB 94-881B). 

Abbreviations and Acronyms
$\mathrm{CCSR}=$ costal cartilage shortening ratio
$\mathrm{CI}=$ clubbing index
$\mathrm{C} / \mathrm{O}=$ calcification/ossification
$\mathrm{CT}=$ computed tomography
RTP = Ravitch thoracoplasty

The operation followed the basic principles of RTP. Subperichondrial removal of the deformed costal cartilages, which were usually associated with ribs 3 through 7 bilaterally, and preservation of the perichondrium were performed. In 5 recent cases, the lateral half of the lower costal cartilages had been preserved. A transverse wedge osteotomy was created at the anterior ( 7 cases) or posterior (27 cases) table of the sternum. No bar or strut was used in any case. One case involved a reoperation owing to recurrence.

CT studies were performed with a 16-slice scanner (Siemens SOMATOM Sensation 16; Siemens, Berlin, Germany) as previously described. ${ }^{1}$

Quantification of the volume of ossification in the costal cartilages was performed on a workstation (GE AW 3.1; Milwaukee, Wis) by removal of the bony thoracic cage at proper window settings. The volume was expressed in cubic centimeters.

Narrowing of the distance between the rib end and the sternum or shortening of the regenerated cartilage was expressed as the costal cartilage shortening ratio (CCSR) and was calculated from the length of regenerated costal cartilage divided by the length of the second costal cartilage (Figure 1). The second rib, which is usually free from surgical exploration in most patients, was used as the reference rib.

We used the clubbing index (CI) to evaluate the severity of the clubbing change at the bony end. The CI was defined as the maximum vertical length at the anterior rib end divided by the vertical length of the same rib at the midaxillary line (Figure 2).

Statistical evaluations were performed with independentsamples $t$ test for CCSR and CI of each rib between RTP patients and controls and by the Pearson correlation coefficient computed by a bivariate correlation procedure for measuring a linear association of $\mathrm{C} / \mathrm{O}$ volume versus age at operation and follow-up, the number of rib removed, and postoperative pectus CT index.

\section{Results}

Thirty-six of the consecutive RTP patients (31 male patients) chose to participate in the follow-up. The mean age was $15.5 \pm 5.32$ years (range: $8.2-26.4$ years) at the time of the follow-up and $6.3 \pm 3.74$ years (range: $2.4-15.6$ years) at the time of the RTP. The average duration between the RTP and the follow-up was $9.08 \pm 4.33$ years (range: 4.4-18.2 years). The control group consisted of 24 patients (16 male patients), and the mean age was 5.2 years (range: 2.3-19.4 years).

Patients rated their satisfaction with the surgical correction as excellent in 5 , good in 13 , fair in 13 , and poor in 5 cases.
Significant differences in the CCSR between the surgical and control groups were present. These differences were observed from the third to sixth ribs on the left $(P<.001)$ and the third to seventh ribs on the right side $(P<.001)$ (Figure 1).

The CI was significantly larger through the third to the fifth ribs bilaterally (Figure 2). No clubbing changes were found in the lower ribs. In the 5 patients whose lateral halves of the costal cartilages were preserved, no clubbing changes were observed at the rib ends.

A histologic specimen, which was the only specimen taken at the RTP reoperation, revealed cancellous bone formation. The $\mathrm{C} / \mathrm{O}$ volume was measured as $5.29 \pm 6.94$ $\mathrm{cm}^{3}$ in the 24 patients with $\mathrm{C} / \mathrm{O}$ change. The volume was significantly correlated with the patients' ages at the time of the RTP $(P=.001)$ and the follow-up $(P<.001)$ (Figure 3$)$. The number of rib cartilages removed and the postoperative pectus CT index was not correlated with the $\mathrm{C} / \mathrm{O}$ volume ( $P=.028$ and.162, respectively). No $\mathrm{C} / \mathrm{O}$ change occurred in the control group.

\section{Discussion}

The central dogma of RTP includes preservation of the perichondrium, resection of deformed cartilages, and regeneration of new cartilages. However, the outcome of the regenerated cartilages was seldom discussed, although scattered descriptive statements about cancellous bone formation after RTP surgery have been made. ${ }^{2,3}$ By using modern CT technology, we detected several findings in the regenerated bones and cartilage after RTP that to our knowledge have not been previously reported.

Unexpectedly, two-thirds of our patients had calcified or ossified regenerated cartilages. Although the occurrence of mineralization and ossification changes of the costal cartilages appears naturally at the end of puberty and increases with age, ${ }^{4-6}$ the postoperative ossification changes that occurred in this series of patients began much earlier than puberty. During the dissection of the perichondrium, which is responsible for the formation of new cartilage, damage to this vulnerable layer could easily take place. In our study, the volume of $\mathrm{C} / \mathrm{O}$ changes was well correlated with the ages at the time of the operation and the follow-up. Patients who were younger at the time of the operation (especially under 4 years old) had less ossification of the cartilage. We speculate that the ossification of regenerated cartilage reflects the limited capability of the perichondrium to produce new cartilage. The presence of cancellous bone in the specimen taken with the RTP reoperation case further supports this speculation.

Several factors might be attributed to the lower CCSR. After the removal of native cartilages, scarring and contraction in the dead space would certainly occur and might shorten the total length of the perichondrium. Without adequate support, the individual bony rib might bend inward at the corresponding 

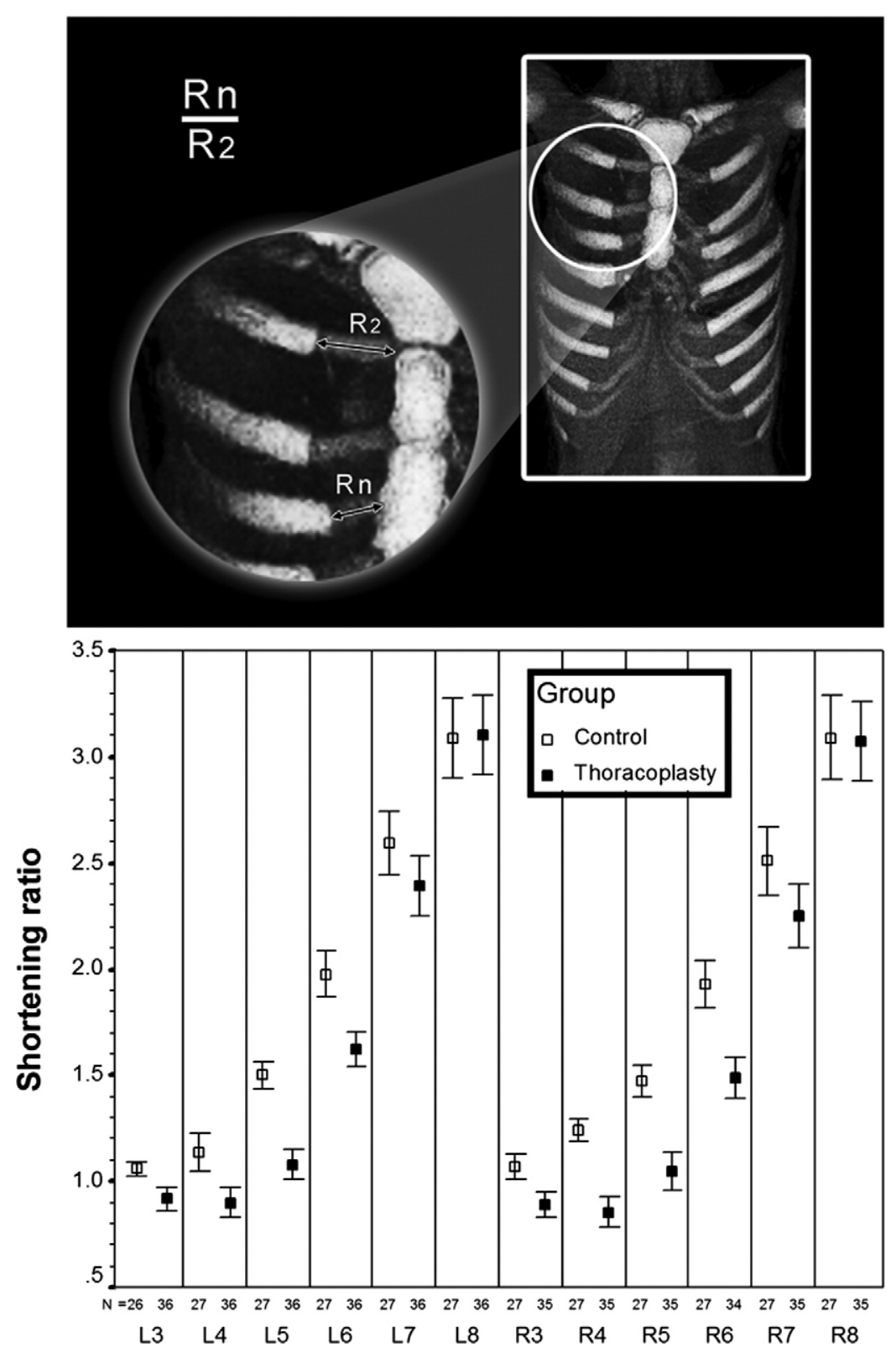

Figure 1. Ln and $R$ n represent the indicated left (L) and right $(R)$ rib number. Inset: Costal cartilage shortening ratio $=R n / R 2$ ( $n$, rib number). level, which would further deteriorate the shortened perichondrium and contribute to a significantly lower CCSR.

Chest wall constriction after RTP was reported by Haller and associates, ${ }^{3}$ and resections of costal cartilages that are both too early and too extensive should be considered regarding this complication. The reason for the restrictive change of the chest wall should not be simply attributed to the $\mathrm{C} / \mathrm{O}$ change of the regenerated cartilage. The present study has shown that the $\mathrm{C} / \mathrm{O}$ changes were smaller when the operation was performed on younger patients. The lowered CSSR, together with the $\mathrm{C} / \mathrm{O}$ changes, might induce the disastrous chest wall constriction. This constriction (or lowered CCSR) was a generalized phenomenon after RTP and could explain why no definite improvement in pulmonary function after RTP is found in the literature. ${ }^{6,7}$

A reaction of subchondral bone associated with a chondral defect and attempted cartilage repair was seen in a goat model. ${ }^{8}$ Resection of the cartilage might induce a reaction in the adjacent bony rib. The healing process from the bony end may be similar to a fracture, and we hypothesized that this process might be the reason for clubbing. Preservation of the costal cartilages adjacent to the bony end of the rib, as observed in our 5 most recent cases, can prevent clubbing of the bony end. This finding again supports our hypothesis.

The CI and CCSR data showed that clubbing changes to the bony end and shortening of the new cartilages all de- 

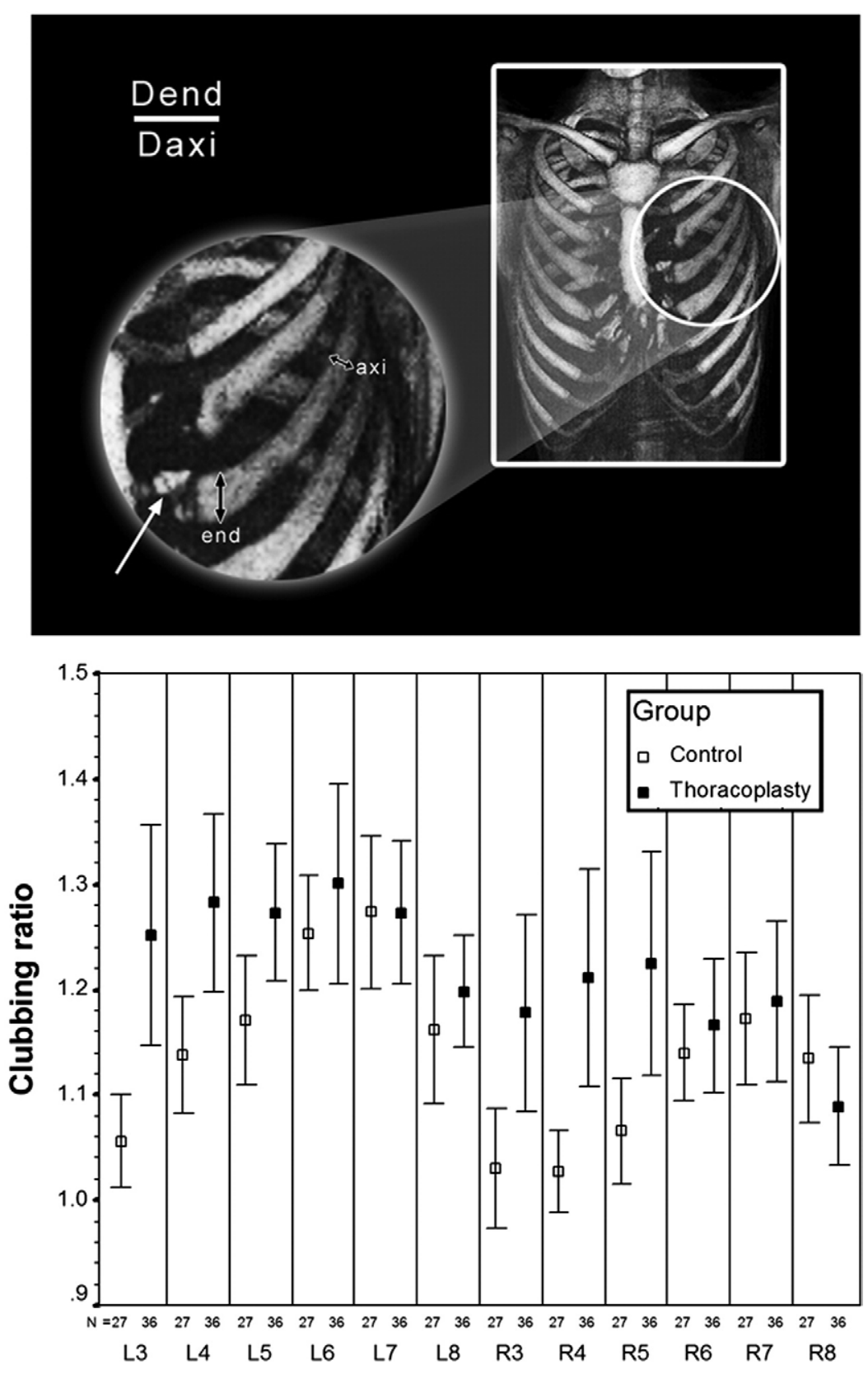

Figure 2. Inset, Clubbing index $=D_{\text {end }} / D_{\text {axi }}$; the maximal vertical length at the anterior rib end $\left(D_{\text {end }}\right) /$ the vertical length of the same rib at the midaxillary line $\left(D_{\text {axi }}\right)$. Calcification/ossification can be found on the regenerated cartilages of the bilateral fourth to seventh ribs. Ossification/calcification is indicated by the arrow. veloped in the third to fifth ribs bilaterally. The ribs in these areas most likely sustained the greatest tensile strength to maintain the contour of the chest wall. Struts were used to prevent the collapse of the chest wall during the regeneration of cartilages and may be crucial to preventing the scarring and contracture from the healing perichondrium, which leads to the decreased CSSR. Preservation of the major portion of the costal cartilages at thoracoplasty might provide more stability to the chest and prevent constriction of the upper chest wall. The routine use of internal support with minimal (or no) cartilage resection was reported to provide excellent long-term results. ${ }^{9-11}$
The use of spiral CT with $3 \mathrm{D}$ reconstruction has been reported in children for preoperative examination and postoperative follow-up by Pretorius and colleagues. ${ }^{12}$ The modern imaging technology offers much better resolution for delineating the reconstructed thoracic cage. The short scanning time of multislice CT with 3-dimensional reconstruction, usually 3 to 4 seconds, is helpful in lessening motion artifacts for better image quality of the bony ribs and costal cartilages, evaluating lung volumes, and gathering more information that we were not able to attain in the past.

In conclusion, the newly developed cartilages from the preserved perichondrium after RTP were different from the 


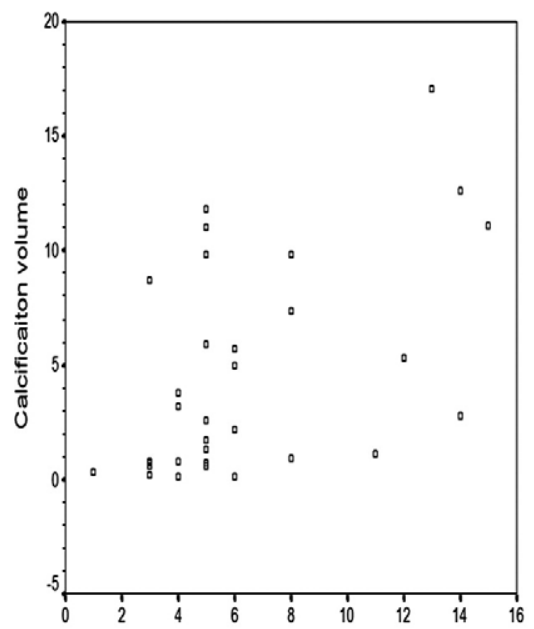

A

Age at operation

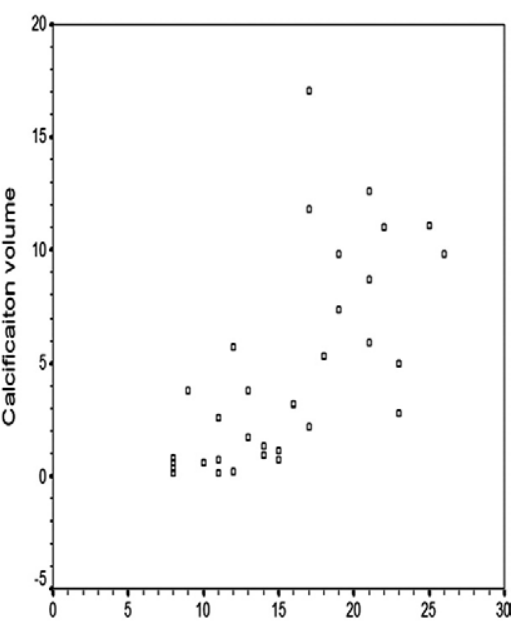

B Age at follow-up
Figure 3. A simple scatter plot of calcification/ ossification (C/O) volume versus patients' ages at the time of the operation (A) and follow-up (B). A linear association was documented by the Pearson correlation coefficient for $\mathrm{C} / 0$ volume versus age at operation $(P=.001)$ and $C / 0$ volume versus age at follow-up $(P<.001)$. native cartilages. The ossification change was significantly correlated with the patient's age at the time of the operation, which might be a reflection of the limited regenerative ability of the perichondrium. Clubbing changes at the bony rib ends and shortening of the regenerated cartilage might be due to the scarring and contractile effect from the healing perichondrium after RTP. Because significant changes were observed in the cartilages and bones after RTP, the use of corrective surgery should be as limited as possible.

\section{References}

1. Chang PY, Lai JY, Chen JC, Wang CJ. Long-term changes of bone and cartilage after Ravitch's thoracoplasty: findings from multi-slice CT with three-dimensional reconstruction. J Pediatr Surg. 2006;41:1947-50.

2. De Ugarte DA, Choi E, Fonkalsrud EW. Repair of recurrent pectus deformities. Am Surg. 2002;68:1075-9.

3. Haller FA, Colombani PM, Humphries CT, Azizkhan RG, Loughlin GM. Chest wall constriction after too extensive and too early operations for pectus excavatum. Ann Thorac Surg. 1996;61:1618-25.

4. Kampen WU, Claassen H, Kirsch T. Mineralization and osteogenesis in the human first rib cartilage. Ann Anat. 1995;177:171-7.

5. Barchiloon V, Hershkovitz I, Rothschild BM, Wish-Baratz S, Latimer B, Jellema LM, et al. Factors affecting the rate and pattern of the first costal cartilage ossification. Am J Forensic Med Pathol. 1996; 17:239-47.

6. Morshuis W, Folgering H, Barentszvan Lier H, Lacquet L. Pulmonary function before surgery for pectus excavatum and at long-term follow-up. Chest. 1994;105:1646-52.

7. Kaguaraoka H, Ohnuki T, Itaoka T, Kei J, Yokoyama M, Nitta S. Degree of severity of pectus excavatum and pulmonary function in preoperative and postoperative periods. J Thorac Cardiovasc Surg. 1992;104:1483-8.

8. Vasara AI, Hyttinen MM, Lammi MJ, Lammi PE, Långsjö TK, Lindahl A, et al. Subchondral bone reaction associated with chondral defect and attempted cartilage repair in goats. Calcif Tissue Int. 2004; 74:107-14.

9. Fonkalsrud EW. Open repair of pectus excavatum with minimal cartilage resection. Ann Surg. 2004;240:231-5.

10. Nuss D, Kelly RE Jr, Croitoru DP, Katz ME. A 10-year review of a minimally invasive technique for the correction of pectus excavatum. J Pediatr Surg. 1998;33:545-52.

11. Croitoru DP, Kelly RE Jr, Goretsky MJ, Lawson ML, Swoveland B, Nuss D. Experience and modification update for the minimally invasive Nuss technique for pectus excavatum repair in 303 patients. J Pediatr Surg. 2002;37:437-45.

12. Pretorius ES, Haller JA, Fishman EK. Spiral CT with 3D reconstruction in children requiring reoperation for failure of chest wall growth after pectus excavatum surgery. Preliminary observations. Clin Imaging. 1998;22:108-16. 